Research Article

\title{
Altered Regional Homogeneity and Functional Connectivity during Microlesion Period after Deep Brain Stimulation in Parkinson's Disease
}

\author{
Bei Luo $\mathbb{D}^{1},{ }^{1}$ Yue Lu $\mathbb{D}$, ${ }^{1}$ Chang Qiu $\mathbb{D}^{1},{ }^{1}$ Wenwen Dong $\mathbb{D}^{1},{ }^{1}$ Chen Xue $\mathbb{D}^{\circ},{ }^{2}$ Dongming Liu $\mathbb{D}^{\circ},{ }^{3}$

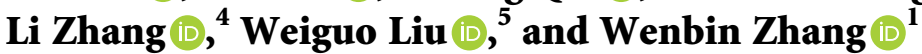 \\ ${ }^{1}$ Department of Functional Neurosurgery, The Affiliated Brain Hospital of Nanjing Medical University, Nanjing 210029, China \\ ${ }^{2}$ Department of Radiology, The Affiliated Brain Hospital of Nanjing Medical University, Nanjing 210029, China \\ ${ }^{3}$ Department of Neurosurgery, The Affiliated Brain Hospital of Nanjing Medical University, Nanjing 210029, China \\ ${ }^{4}$ Department of Geriatrics, The Affiliated Brain Hospital of Nanjing Medical University, Nanjing 210029, China \\ ${ }^{5}$ Department of Neurology, The Affiliated Brain Hospital of Nanjing Medical University, Nanjing 210029, China
}

Correspondence should be addressed to Wenbin Zhang; wenbinzhang@njmu.edu.cn

Received 17 May 2021; Revised 10 August 2021; Accepted 23 August 2021; Published 2 September 2021

Academic Editor: Hélio Teive

Copyright $(\odot 2021$ Bei Luo et al. This is an open access article distributed under the Creative Commons Attribution License, which permits unrestricted use, distribution, and reproduction in any medium, provided the original work is properly cited.

\begin{abstract}
Background. Patients with Parkinson's disease (PD) undergoing deep brain electrode implantation experience a temporary improvement in motor symptoms before the electrical stimulation begins. We usually call this the microlesion effect (MLE), but the mechanism behind it is not clear. Purpose. This study aimed to assess the alterations in brain functions at the regional and whole-brain levels, using regional homogeneity ( $\mathrm{ReHo}$ ) and functional connectivity (FC), during the postoperative microlesion period after deep brain stimulation (DBS) in PD patients. Method. Resting-state functional MRI data were collected from 27 PD patients before and after the first day of DBS and 12 healthy controls (HCs) in this study. The ReHo in combination with FC analysis was used to investigate the alterations of regional brain activity in all the subjects. Results. There were increased ReHo in the basal ganglia-thalamocortical circuit (left supplementary motor area and bilateral paracentral lobule), whereas decreased ReHo in the default mode network (DMN) (left angular gyrus, bilateral precuneus), prefrontal cortex (bilateral middle frontal gyrus), and the cerebello-thalamocortical (CTC) circuit (Cerebellum_crus2/1_L) after DBS. In addition, we also found abnormal FC in the lingual gyrus, cerebellum, and DMN. Conclusion. Microlesion of the thalamus caused by electrode implantation can alter the activity of the basal ganglia-thalamocortical circuit, prefrontal cortex, DMN, and CTC circuit and induce abnormal FC in the lingual gyrus, cerebellum, prefrontal cortex, and DMN among PD patients. The findings of this study contribute to the understanding of the mechanism of MLE.
\end{abstract}

\section{Introduction}

Deep brain stimulation (DBS) is a widely accepted surgical method used for the treatment of mid-to-late-stage Parkinson's disease (PD), which can significantly improve motor symptoms of $\mathrm{PD}$ and reduce drug-induced complications [1-3]. Currently, the subthalamic nucleus (STN) and internal pallidum (GPi) are commonly targeted by DBS in the treatment of PD [4-6]. After DBS electrodes are implanted, a temporary improvement in the motor symptoms of PD patients, including tremor, rigidity, and bradykinesia, can be observed immediately over several days to weeks. We usually call this the microlesion effect (MLE). Many studies have reported this [7-9]. However, the mechanisms underlying the improvement in motor symptoms are not well understood. In addition, previous studies have also suggested that postoperative verbal fluency $[3,10]$ and cognitive decline $[3,10]$ may be related to MLE. Some studies have found that MLE is likely associated with damage to peripheral neurons or glial cells, with subsequent degeneration loss, released neurotransmitters of local neurons, and edema around the electrode trajectory through the implantation of electrodes $[7,8]$.

Resting-state functional magnetic resonance imaging (rs-fMRI) is a type of brain MRI scan conducted while the 
subject remains motionless, closes his/her eyes, and does not carry out any active thinking in a calm state of awakening. Compared to the task state fMRI, rs-fMRI is simple and highly repeatable. It has been widely applied to investigate the alterations of brain activity in patients with neurological and mental illnesses [11-13]. The regional homogeneity [14] (ReHo) is a data-processing method proposed by Zang et al. in 2004 to analyze rs-fMRI data. The ReHo method indirectly reflects the spontaneous activity of neurons by analyzing the consistency of signaling fluctuations in the spatial adjacent voxel oxygen levels simultaneously using Kendall's coefficient of concordance (KCC) [14]. Reho has been widely used to study abnormalities in brain activity in PD [15-17] and can even be used as a potential imaging marker for PD $[15,18]$. Conducting the ReHo method of the entire gray mass of the brain has almost shown test-retest reproducibility [19]. Functional connectivity (FC) is an fMRI dataprocessing method used to study brain networks to detect the correlations of spontaneous activity between different brain regions.

Currently, there are no literature reports on the use of ReHo, combined with the FC method, to study brain function during the postoperative microlesion phase of DBS. Therefore, the present study explored the functional changes of the brain region during the microlesion period by ReHo and FC analysis using the rs-fMRI whole-brain data.

\section{Materials and Methods}

2.1. Participants and Clinical Assessments. We initially recruited $34 \mathrm{PD}$ patients for this study. All PD diagnoses were based on the UK Brain Bank Criteria for the diagnosis of PD in 1992 [20]. All PD patients met the indications for DBS surgery and underwent DBS surgery. The exclusion criteria included the following: (1) patients with a nonprimary Parkinson's disease diagnosis or an unknown Parkinson's disease diagnosis; (2) other central nervous system diseases or disorders (i.e., encephalitis, brain tumor, epilepsy); (3) drugs that affect brain function, such as antipsychotics and antidepressants; (4) contraindications for MRI scanning. In addition, 12 healthy controls (HCs) (six males and six females) with matched gender and age were recruited from the patients' family members and the community during the same period. All the subjects were right-handed. We used the Hamilton Anxiety (HAMA) and Hamilton Depression (HAMD) scales to evaluate the mental and psychological statuses of the participants. The overall cognitive level was assessed using the Montreal Cognitive Assessment (MoCA). The 39-item Parkinson's Disease Questionnaire (PDQ-39) was utilized to assess the quality of life in patients with PD. Furthermore, the patients' motor symptoms were assessed with the Unified Parkinson's Disease Rating Scale part-III (UPDRS-III). Verbal fluency (VF) was used to assess the patients' ability to produce words for a limited time and the patients' verbal memory. In this test, $\mathrm{PD}$ patients were asked to name as many animals as possible in 60 seconds, which is a semantic fluency test. Trail Making Test (TMT, part A, B) was used to evaluate the executive function of patients, and Benton judgment of line orientation test (BJLOT) was used to evaluate the visuospatial function of patients. In this study, the patients were tested using the above scales, including MoCA, UPDRS-III, VF, TMT, and BJLOT in three sessions: before DBS, one day after DBS, and one month after DBS. Preoperative $\mathrm{PD}$ data were included in the pre-PD group, and postoperative data were included in the post-PD group. The scale and MRI data of PD patients were collected 12 hours after drug disuse and before electrical stimulation to exclude the interference of drug and electrical stimulation to data acquisition. The study obtained the approval of the Ethics Committee of The Brain Hospital affiliated with Nanjing Medical University and written informed consent from all subjects.

2.2. Surgical Procedure. All PD patients who perform DBS surgery chose bilateral STN as the surgical target. Leksell planning system software was used to fuse the $3.0 \mathrm{~T}$ head MRI scan data and CT scan data after the head positioning frame. The midpoint of the anterior commissure (AC) and posterior commissure (PC) is used as the origin to establish the coordinate system, and the surgical plan was made. The STN target was located $11-12 \mathrm{~mm}$ beside the origin, $3 \mathrm{~mm}$ backward, and $4 \mathrm{~mm}$ downward. In addition, the target coordinates were fine-tuned according to the specific position of the STN nucleus in the MRI image. The arc and ring angles of the implanted path were determined by designing a cranial approach that avoided cerebral surface vessels, sulci, and lateral ventricles. The operation was performed under a combination of local and general anesthesia. The preoperative plan was strictly followed during the electrode implantation, and the electrode position was not adjusted during and after the operation. DBS surgery was performed by a single neurosurgeon using a consistent surgical procedure. Postoperative CT and MRI reexaminations revealed no serious surgical complications except a small amount of intracranial gas in some patients.

2.3. Image Acquisition. MRI examinations were performed with a 1.5 Tesla GE Medical Systems scanner via an 8channel head coil. The participants were asked to keep quiet, close their eyes, stay awake, and not think or carry out any voluntary movements on the examination bed during the scanning process. The patients were scanned before and after the first day of DBS, while the HCs group was scanned once. The imaging data were collected by a gradient-recalled echoplanar imaging (GRE-EPI) sequence with the following parameters: repetition time $(\mathrm{TR})=2000 \mathrm{~ms}$, echo time $(\mathrm{TE})=40 \mathrm{~ms}$, slice number $=28$, thickness $=3.0 \mathrm{~mm}$ with no gap, flip angle $(\mathrm{FA})=90^{\circ}$, field of view $(\mathrm{FOV})=240 \times$ $240 \mathrm{~mm}$, matrix size $=64 \times 64$, voxel size $=3.75 \times 3.75 \times 3$ $\mathrm{mm}^{3}$, and 128 total volumes. The $\mathrm{T} 1$-weighted data were collected by a 3D magnetization-prepared rapid gradientecho (MPRAGE) sequence with $\mathrm{TR}=11.864 \mathrm{~ms}, \mathrm{TE}=4.932$ $\mathrm{ms}, \mathrm{FA}=20^{\circ}$, matrix size $=256 \times 256, \mathrm{FOV}=152 \times 152 \mathrm{~mm}$, thickness $=1.4 \mathrm{~mm}$, number of slices $=112$, and voxel size $=0.59 \times 0.59 \times 1.4 \mathrm{~mm}^{3}$. 
2.4. Data Preprocessing. The data preprocessing was performed using the rs-fMRI data-processing assistant (DPABI4.3, http://rfmri.org/dpabi) on the MATLAB 2013b platform (https://www.mathworks.com/products/matlab). The resting-state data were preprocessed using the following steps: (1) disregard the first five time points; (2) slice timing correction; (3) head motion correction (seven patients with a head translation $>3.0 \mathrm{~mm}$ and rotation $>3.0^{\circ}$ in any direction were excluded); (4) spatial normalization to the standard Montreal Neurological Institute (MNI) template and resampled to $3 \times 3 \times 3 \mathrm{~mm}^{3}$; (5) removed the linear trend and time-bandpass filter $(0.01 \mathrm{~Hz} f<0.10 \mathrm{~Hz})$. The nuisance variables, including 24 motion parameters, global signals, white matter signal, and cerebrospinal fluid signal, were regressed using the linear regression analysis. Spatial smoothing was conducted in preprocessing the FC analysis but not in ReHo analysis.

2.5. ReHo and FC Analysis. The filtering data without smoothing was utilized to conduct ReHo analysis in the DPABI software to calculate the consistency of each voxel and its adjacent 26 voxels in time series, which was the ReHo value of the voxel. The standardization of ReHo was obtained by dividing the KCC value of the individual voxel by the mean KCC of the whole brain. Finally, spatial smoothing was applied to the ReHo maps with a Gaussian kernel with full width at half maximum of $4 \times 4 \times 4 \mathrm{~mm}$ to further improve the image signal-to-noise ratio and decrease the errors caused by the spatial standardization process.

Brain regions with significant differences in ReHo values between the pre-PD group and post-PD group were defined as areas of interest (ROIs). FC were analyzed using a seedbased approach through the resting-state fMRI data analysis toolkit (http://restfmri.net/forum/REST). Finally, all FC maps are normalized by Fisher's z-transformation to improve the normality of data distribution.

2.6. Statistical Analysis. The two-sample $t$-test and chisquared test were used to compare differences in demographic and clinical data between groups, respectively. In addition, the MoCA, UPDRS-III, VF, TMT, and BJLOT scores across different sessions in PD patients were compared using a repeated measures analysis of variance and post hoc tests were used between every two sessions. The statistically significant threshold was set to 0.05 . All the above comparisons were performed using SPSS22 (Chicago, IL, USA) software.

The two-sample $t$-test was conducted to compare differences of ReHo value between the pre-PD group and HCs with age, sex, and mean framewise displacement (FD) as covariates in SPM12 (https://www.fil.ion.ucl.ac.uk/spm/ software/spm12/). The data of the pre-PD and post-PD groups were rs-fMRI data obtained from the same group of patients at different time intervals. Therefore, the differences of $\mathrm{ReHo} / \mathrm{FC}$ between the pre-PD and post-PD groups were assessed using paired $t$-tests with mean FD as a covariate. $P<0.001$ at voxel level was used as the threshold of statistical significance. The threshold of statistical significance was set at $P<0.001$. Multiple comparisons of the family-wise error rate with cluster $P<0.05$ were applied to correct for excluding false-positive results.

\section{Results}

3.1. Demographic and Clinical Features. Twenty-seven patients (11 males and 16 females) were included in the PD group after excluding six patients, while 12 subjects (six males and six females) were recruited as the healthy controls HCs. The demographic and clinical characteristics of the subjects are presented in Table 1 . PD patients and HCs did not differ in gender $(P=0.59)$ and age $(P=0.60)$. There was a significant difference in MoCA $(P<0.001)$, HAMA $(P<0.001)$, and HAMD $(P<0.001)$ between PD patients and HCs. The MoCA $(P=0.001)$, UPDRS-III $(P<0.001)$, VF $(P<0.001)$, TMT $(P<0.001)$, and BJLOT $(P<0.001)$ scores for $\mathrm{PD}$ patients varied significantly over three sessions. In all the post hoc comparisons, we found significant differences $(P<0.001)$ between every two sessions of each scale except for MoCA before DBS and one month after DBS in $\mathrm{PD}$ patients $(P=0.097)$.

3.2. Altered ReHo. Relative to the HCs, the pre-PD group indicated increased $\mathrm{ReHo}$ in the right superior frontal gyrus (SFG), while they exhibited decreased ReHo in the right middle frontal gyrus (MFG), right middle temporal gyrus (MTG), right superior occipital gyrus (SOG), right middle occipital gyrus (MOG), and right angular gyrus (shown in Table 2 and Figure 1). After DBS, the post-PD group demonstrated increased ReHo in multiple brain regions, including the right inferior frontal gyrus (IFG), left supplementary motor area (SMA), left median cingulate and paracingulate (DCG) gyri, and bilateral paracentral lobule (PCL) compared to the pre-PD group. In addition, the post$\mathrm{PD}$ group exhibited decreased ReHo in the left posterior cerebellar lobe (Cerebellum_crus2/1_L), left MTG, left angular gyrus, bilateral precuneus, and bilateral MFG compared to the pre-PD group (shown in Table 2 and Figure 2).

3.3. Altered FC. Brain regions that showed significant differences in ReHo between the pre-PD and post-PD groups were eventually defined as ROIs. These brain regions include the right IFG, left SMA, PCL, left posterior cerebellar lobe, left MTG, precuneus, left MFG, and right MFG. Then we calculated the correlation between the seeds and the wholebrain voxel in a voxel-wise approach. There were no significant intergroup differences in FC for the two ROIs (right IFG and left SMA). The post-PD group demonstrated significantly reduced precuneus FC in the default mode network (DMN) (angular, precuneus, and superior frontal gyrus) compared to the pre-PD group. Similarly, we found reduced FC between the bilateral MFG and DMN. However, the post-PD group exhibited increased precuneus FC with right SMA and right supramarginal gyrus. In addition, the post-PD group had higher FC of left MFG with left supramarginal gyrus, bilateral cerebellum, and bilateral lingual gyrus. Furthermore, there was increased right MFG 
TABle 1: Demographic and clinical data of all subjects.

\begin{tabular}{|c|c|c|c|}
\hline & HCs $(n=12)$, mean \pm SD & $\mathrm{PD}(n=27)$, mean $\pm \mathrm{SD}$ & $P$ value \\
\hline Age (years) & $63.92 \pm 8.38$ & $62.30 \pm 8.97$ & $0.60^{\mathrm{a}}$ \\
\hline Sex (male/female) & $6 / 6$ & $16 / 11$ & $0.59^{\mathrm{b}}$ \\
\hline Disease duration (year) & NA & $8.26 \pm 2.78$ & - \\
\hline $\operatorname{LEDD}(\mathrm{mg} / \mathrm{d})$ & NA & $765.78 \pm 176.74$ & - \\
\hline HAMA score & $0.42 \pm 0.51$ & $5.59 \pm 3.71$ & $<0.001^{\mathrm{a} *}$ \\
\hline HAMD score & $0.75 \pm 0.87$ & $5.63 \pm 3.95$ & $<0.001^{\mathrm{a} *}$ \\
\hline PDQ-39 score & NA & $42.96 \pm 16.97$ & - \\
\hline \multicolumn{4}{|l|}{ MoCA score } \\
\hline Before DBS & $28.67 \pm 1.07$ & $24.22 \pm 3.94$ & $<0.001^{\mathrm{a} *}$ \\
\hline One day after DBS & NA & $21.82 \pm 4.78$ & - \\
\hline One month after DBS & NA & $23.78 \pm 3.81$ & $0.001^{\mathrm{c} *}$ \\
\hline \multicolumn{4}{|l|}{ UPDRS-III score } \\
\hline Before DBS & NA & $37.70 \pm 12.04$ & - \\
\hline One day after DBS & NA & $27.52 \pm 6.98$ & - \\
\hline One month after DBS & NA & $38.70 \pm 10.89$ & $<0.001^{\mathrm{C} *}$ \\
\hline \multicolumn{4}{|l|}{ VF score } \\
\hline Before DBS & NA & $20.63 \pm 4.51$ & - \\
\hline One day after DBS & NA & $13.85 \pm 4.10$ & - \\
\hline One month after DBS & NA & $16.30 \pm 4.03$ & $<0.001^{\mathrm{c} *}$ \\
\hline \multicolumn{4}{|l|}{ BJLOT score } \\
\hline Before DBS & NA & $21.30 \pm 2.74$ & - \\
\hline One day after DBS & NA & $16.85 \pm 3.21$ & - \\
\hline One month after DBS & NA & $19.48 \pm 2.83$ & $<0.001^{\mathrm{c}_{*}}$ \\
\hline \multicolumn{4}{|l|}{ TMT-A score } \\
\hline Before DBS & NA & $45.70 \pm 12.08$ & - \\
\hline One day after DBS & NA & $52.89 \pm 11.62$ & - \\
\hline One month after DBS & NA & $49.96 \pm 11.33$ & $<0.001^{\mathrm{c} *}$ \\
\hline \multicolumn{4}{|l|}{ TMT-B score } \\
\hline Before DBS & NA & $99.07 \pm 10.96$ & - \\
\hline One day after DBS & NA & $109.22 \pm 10.93$ & - \\
\hline One month after DBS & NA & $103.74 \pm 11.06$ & $<0.001^{\mathrm{C}_{*}}$ \\
\hline
\end{tabular}

HCs, healthy controls; PD, Parkinson's disease; LEDD, levodopa equivalent daily dose; MoCA, Montreal cognitive assessment; HAMA, Hamilton anxiety scale; HAMD, Hamilton depression scale; UPDRS-III, the unified Parkinson's disease rating scale part-III; PDQ-39, the 39-item Parkinson's disease questionnaire; VF, verbal fluency; BJLOT, Benton judgment of line orientation test; TMT-A/B, trail making test part A/B; NA, not applicable; DBS, deep brain stimulation; $\mathrm{SD}$, standard deviation. ${ }^{\mathrm{a}} P$ value was obtained by a two-sample $t$-test between $\mathrm{PD}$ and HCs groups. ${ }^{\mathrm{b}} P$ value was obtained by a chi-square test between PD and HCs groups. ${ }^{c}$ Pvalue was obtained by a repeated measures ANOVA test in three sessions. ${ }^{*} P<0.05$.

FC with left IFG and left supramarginal gyrus in the post-PD group. In addition, the post-PD group exhibited higher FC of PCL with right DCG compared with the pre-PD group. The above findings are presented in Table 3 and Figure 3.

\section{Discussion}

Herein, we used ReHo, combined with the FC method, to explore differences in brain function in resting state in PD patients during the microlesion period after DBS. The microlesion caused by implantation of the lone DBS electrodes can cause changes in the brain activity patterns and brain network. The increase or decrease in ReHo reflects increased or decreased consistency of spontaneous activity of neurons within the local brain region. Alterations in FC provide a basis for functional network reorganization of brain regions. After DBS surgery, the UPDRS-III score decreased significantly from $37.70 \pm 12.04$ to $27.52 \pm 6.98$, consistent with improved motor symptoms observed after surgery. After one month, the score deteriorated to $38.70 \pm 10.89$, reflecting the transient characteristics of symptom improvements during the microlesion period. In addition, on day one after surgery, the overall cognitive level, verbal fluency, visuospatial function, and executive function decreased according to the scale scores, suggesting that the microlesion of the implant pathway impaired these functions. One month after surgery, the VF performance, visuospatial function, and executive function were partially restored. However, the patients' overall cognitive level was also elevated to the preoperative level, suggesting that microlesion of the implant trajectory caused only a temporary decline in the patients' overall cognition, with no significant long-term impact, similar to a previous study. This study suggested that although STN-DBS selectively reduced frontal cognitive functions, it did not reduce patients' overall cognition or ability [21].

This study found that the ReHo value in the DMN-related region (right angular gyrus and right $\mathrm{MFG}$ ) decreased in PD patients compared to HCs, suggesting that the DMN was partially impaired in $\mathrm{PD}$, similar to results of previous studies [22, 23]. Previous studies reporting the brain region of alterations of ReHo in PD patients were not precisely 
TABLE 2: Regions with ReHo differences between the pre-PD group and HCs, as well as between the pre-PD group and the post-PD group (voxel $P<0.001$, FWE correction with cluster $P<0.05$ ).

\begin{tabular}{|c|c|c|c|c|c|c|}
\hline & \multirow{2}{*}{ Brain region $(\mathrm{AAL})$} & \multirow{2}{*}{ Voxel size } & \multicolumn{3}{|c|}{ MNI coordinate } & \multirow{2}{*}{ Peak $T$-value } \\
\hline & & & $X$ & $Y$ & $Z$ & \\
\hline \multicolumn{7}{|l|}{ Pre-PD>HC } \\
\hline Cluster1 & Frontal_Sup_Orb_R & 47 & 15 & 39 & -30 & 4.6419 \\
\hline \multicolumn{7}{|l|}{$H C>$ pre- $P D$} \\
\hline Cluster1 & Frontal_Mid_R & 39 & 45 & 42 & 15 & -5.9629 \\
\hline Cluster2 & Temporal_Mid_R & 75 & 60 & -54 & 12 & -4.7052 \\
\hline Cluster3 & Occipital_Sup_R & 51 & 24 & -87 & 18 & -5.5474 \\
\hline Cluster4 & Occipital_Mid_R & 47 & 33 & -60 & 27 & -4.325 \\
\hline Cluster5 & Angular_R & 62 & 27 & -66 & 42 & -5.0054 \\
\hline \multicolumn{7}{|c|}{ Pre- $P D>$ post $-P D$} \\
\hline Cluster1 & Cerebellum_Crus1/2_L & 65 & -39 & -45 & -45 & 4.8602 \\
\hline Cluster2 & Temporal_Mid_L/angular_L & 48 & -60 & -60 & 15 & 11.8391 \\
\hline Cluster3 & Precuneus_R/L & 88 & 0 & -60 & 42 & 4.9435 \\
\hline Cluster4 & Frontal_Mid_L & 167 & -27 & 24 & 51 & 7.2589 \\
\hline Cluster5 & Frontal_Mid_R & 62 & 39 & 30 & 54 & 5.6951 \\
\hline \multicolumn{7}{|c|}{ Post-PD $>$ pre-PD } \\
\hline Cluster1 & Frontal_Inf_Orb_R & 35 & 24 & 18 & -21 & -5.3848 \\
\hline Cluster2 & Supp_Motor_Area_L/cingulum_Mid_L & 51 & -9 & -9 & 45 & -7.0682 \\
\hline Cluster3 & Paracentral_Lobule_R/L & 59 & -3 & -15 & 78 & -5.5623 \\
\hline
\end{tabular}

HCs, healthy controls; PD, Parkinson's disease; ReHo, regional homogeneity; FWE, the family-wise error; pre-PD, before DBS; post-PD, one day after DBS; AAL, anatomical automatic labeling; MNI, Montreal Neurological Institute; DBS, deep brain stimulation.

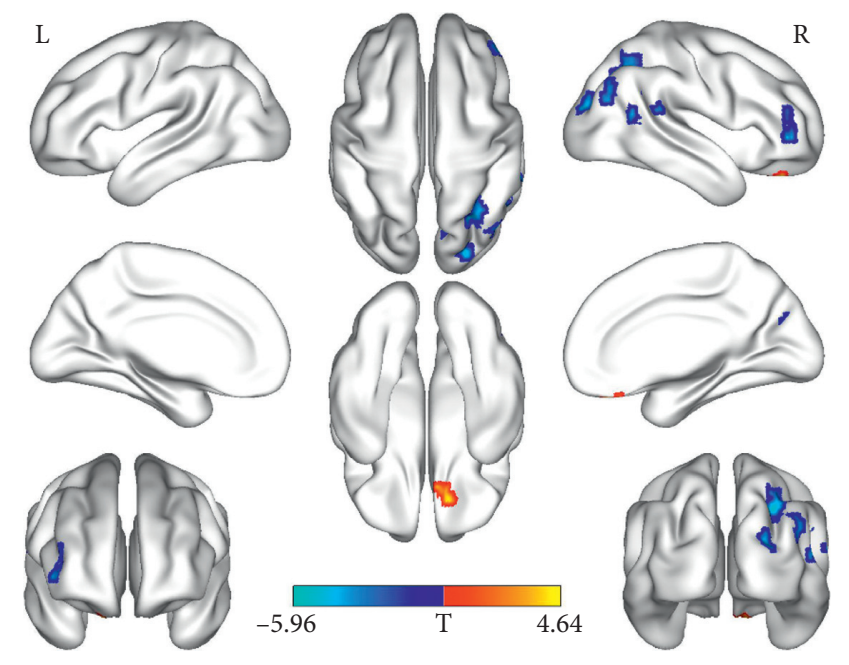

(a)

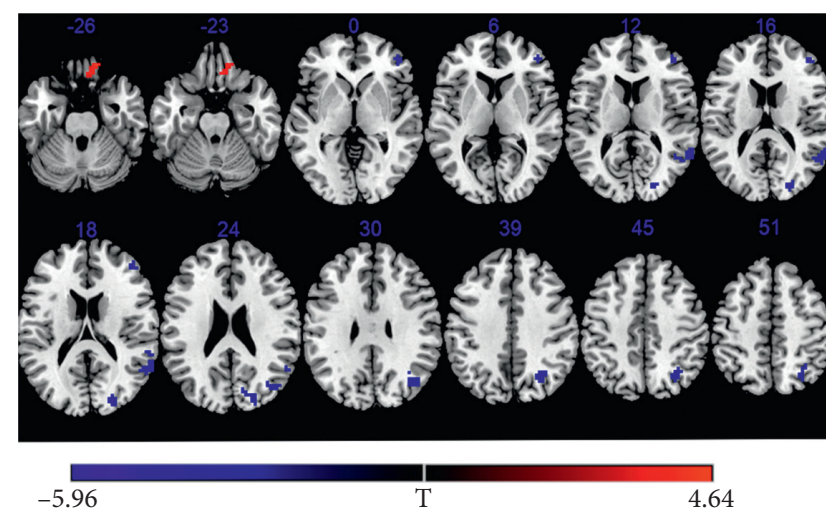

(b)

Figure 1: Regions showing ReHo differences between the pre-PD group and HCs (voxel $P<0.001$, FWE correction with cluster $P<0.05$ ). Regions showing increased ReHo in red and decreased ReHo in blue; surface (a) and transverse (b) views are shown; L, left hemispheres; R, right hemispheres; FWE, multiple comparisons of the family-wise error (voxel $P<0.001$, cluster $P<0.05$ ); ReHo, regional homogeneity; pre-PD, before DBS; post-PD, one day after DBS; PD, Parkinson's disease; DBS, deep brain stimulation; HCs, healthy controls.

consistent with this study, which might be attributed to the different subtypes and disease severity of PD patients included in this study. Many studies have consistently suggested that DMN plays an important role in cognitive processing [22-24]. Hou et al. [24] suggested that changes in DMN connectivity were characteristic of PD with a mild cognitive impairment. However, in a study on PD patients with unimpaired cognitive ability, the function of DMN was damaged, suggesting that the impairment of DMN had already occurred in $\mathrm{PD}$ patients before cognitive impairment [22]. In addition, a decreased ReHo value in the visual association cortex (right SOG and right MOG) was found in PD patients, consistent with prior imaging studies demonstrating reduced occipital leaf perfusion or metabolism in PD $[25,26]$.

We observed a higher ReHo value in the mesial premotor region (left SMA) and the primary motor cortex (M1), including in the bilateral PCL after deep brain electrodes implantation in the post-PD group relative to the pre-PD group, similar to the discovery of a previous study that focused on brain regions activated during the tapping test for microlesion [8]. SMA and M1 are important components 


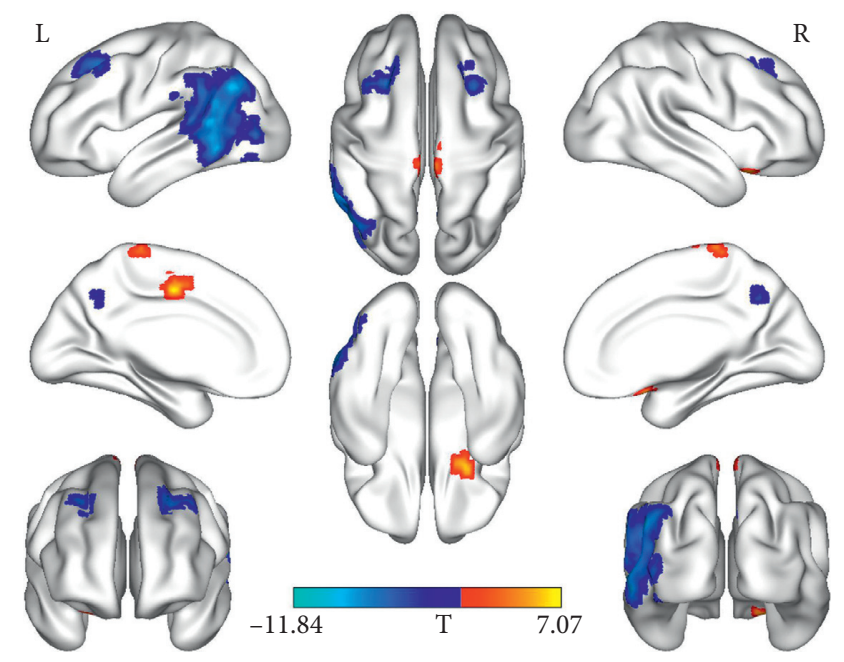

(a)

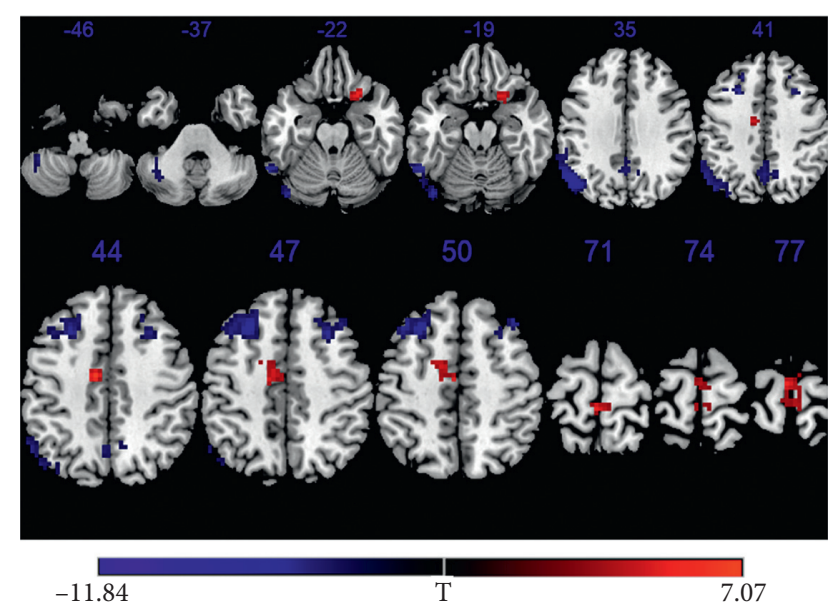

(b)

Figure 2: Regions showing ReHo differences between the pre-PD group and the post-PD group (voxel $P<0.001$, FWE correction with cluster $P<0.05)$. Regions showing increased ReHo in red and decreased ReHo in blue; surface (a) and transverse (b) views are shown; L, left hemispheres; R, right hemispheres; FWE, multiple comparisons of the family-wise error (voxel $P<0.001$, cluster $P<0.05$ ); ReHo, regional homogeneity; pre-PD, before DBS; post-PD, one day after DBS; PD, Parkinson's disease; DBS, deep brain stimulation; HCs, healthy controls.

of the basal ganglia-thalamocortical circuit. Some studies have consistently revealed that PD patients have less SMA activation or reduced activity [27-30], believed to be caused by damage to dopaminergic neurons in the basal ganglia of PD patients [31]. Haslinger et al. [28] reported that changes in SMA activity were highly associated with motor ability. After levodopa treatment, the activity of the SMA region increased, inducing relative normalization of impaired activation of the premotor cortex in PD patients [28, 31]. One study found that one-sided pallidotomy significantly increased the activity of SMA in PD patients during voluntary activity [32], consistent with the present study; however, the slight damage due to DBS surgery is less than that of pallidotomy.

Therefore, we believe that the increased activity of SMA after electrode implantation induces relative normalization of impaired mesial premotor regions in PD patients. The structure and function of SMA make this cortex region a possible target for neuromodulation therapy. M1 is the area responsible for motion output, and previous studies have demonstrated decreased M1 activity when PD patients exercise [33]. Kann et al. [34] reported a decrease in the volume of gray matter in the PCL and motor cortex associated with symptoms in the rigid motor subtype of PD. A previous study discovered decreased cortical thickness of PCL and motor cortex in PD patients with gait disorders [35]. Herein, it is believed that the increased ReHo of SMA and PCL in the microlesion period can relatively normalize the function of the motor-related brain areas and improve the neural activity of the basal ganglia-thalamocortical circuit, causing an improvement in postoperative symptoms in patients. The findings of this study are similar to those of a recently published paper [36]. Yue et al. used amplitude of lowfrequency fluctuation (ALFF) to analyze the differences in brain activity before and after DBS surgery found that the ALFF value of the precentral gyrus decreased after surgery. $\mathrm{ReHo}$ revealed the neural synchronization of local brain regions activity, while ALFF reflected the spontaneous neural activity of local brain regions [16]. ALFF combined with the ReHo method can reflect the pattern of neural activity more comprehensively. The mechanism of surgical damage has been further elucidated by exploring the mechanism of MLE.

After DBS surgery, we observed decreased ReHo in DMN-related areas (left angular gyrus, bilateral precuneus) and the CTC circuit (Cerebellum_Crus2/1_L). A previous study of microlesion also showed decreased activity in DMN-related brain regions after DBS [36]. However, they did not assess executive function or speech function after DBS. The DMN is a system that is active during rest and is closely related to cognition. Several studies have discovered that after STN-DBS implantation, the patients' cognitive aspects, particularly verbal fluency $[3,10]$, decrease, which is caused by MLE [3, 21, 37]. One study showed a continuous decline in VF performance in the case of nonelectrical stimulation, indicating that it is mainly due to surgical damage instead of electrical stimulation [38]. Meanwhile, the post-PD group showed decreased ReHo values in bilateral MFG relative to the pre-PD group. The MFG is a key region of executive function, involved in cognitive processing, emotional regulation, and working memory [39-41], consistent with the decline in cognitive level, VF performance, and executive function observed in patients after surgery in this study.

Surprisingly, further FC analysis in these regions revealed that the post-PD group had a similar decline with DMN-related area and prefrontal cortex compared to the pre-PD group. The results of the present study once again 
TABLE 3: Regions showing altered functional connectivities between the pre-PD group and the post-PD group (voxel $P<0.001, \mathrm{FWE}$ correction with cluster $P<0.05$ ).

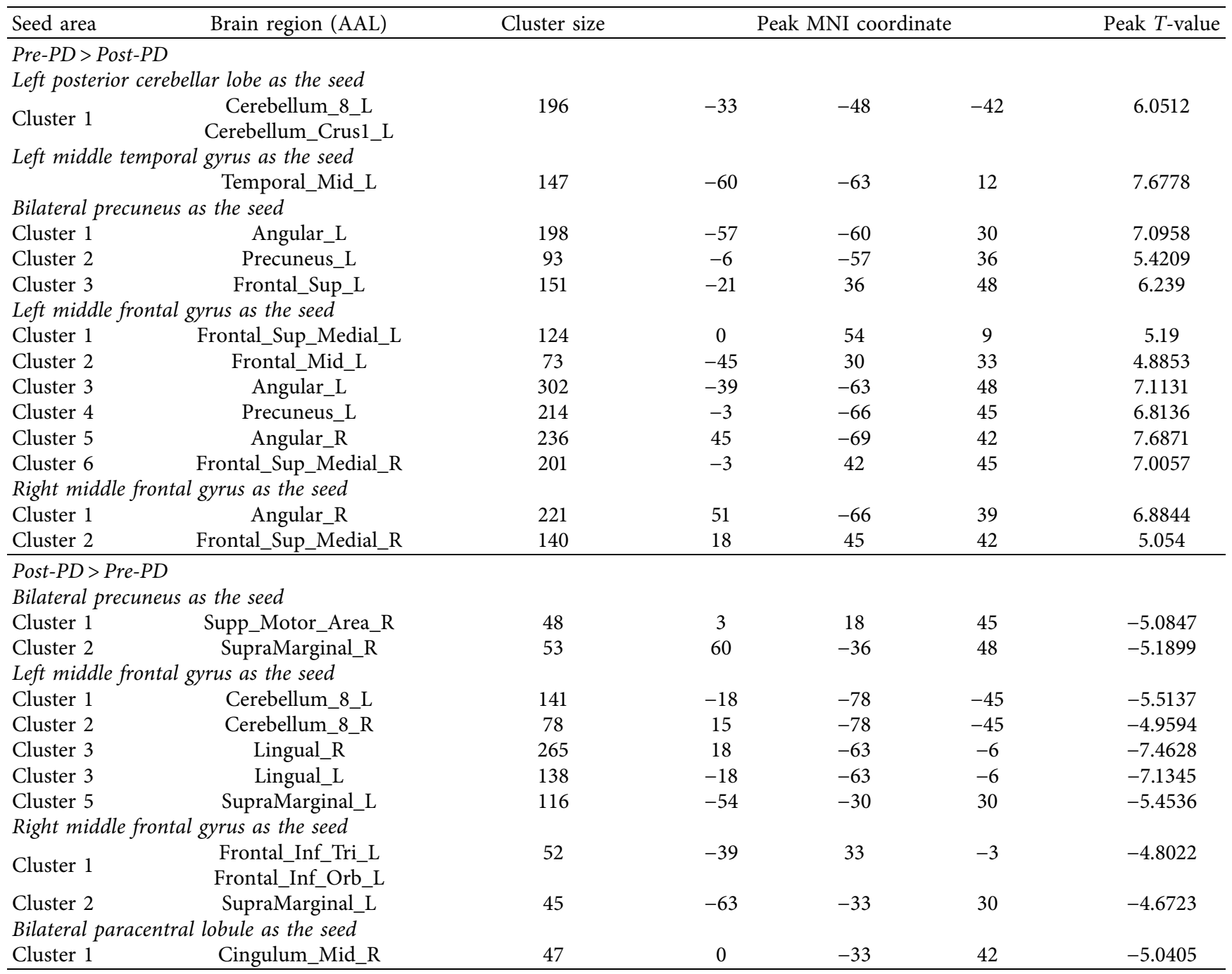

Pre-PD, before DBS; post-PD, one day after DBS; AAL, anatomical automatic labeling; MNI, Montreal Neurological Institute; PD, Parkinson's disease; DBS, deep brain stimulation; FWE, the family-wise error.

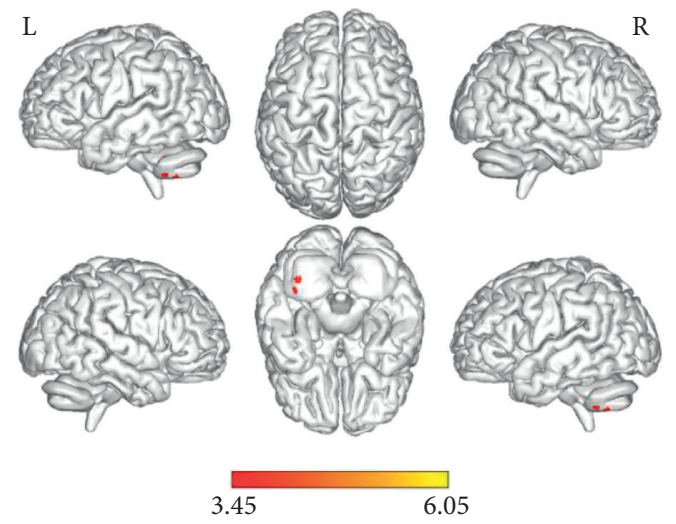

(a)

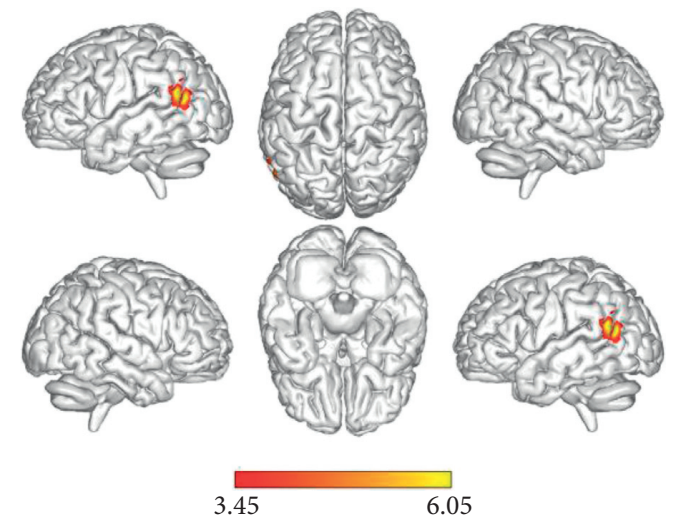

(b)

Figure 3: Continued. 


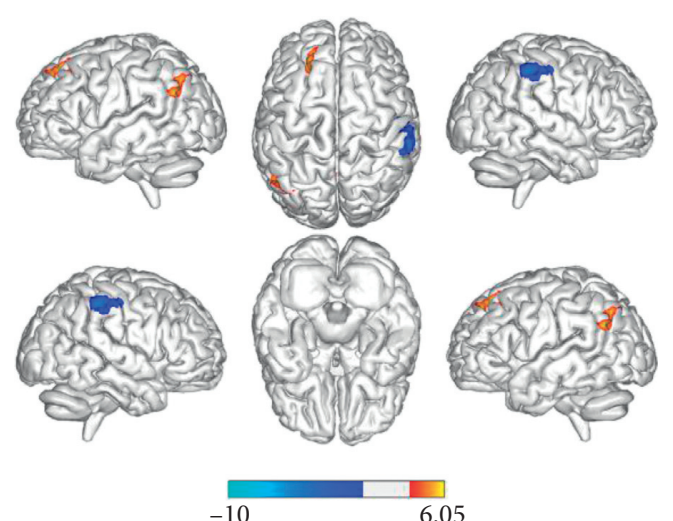

(c)

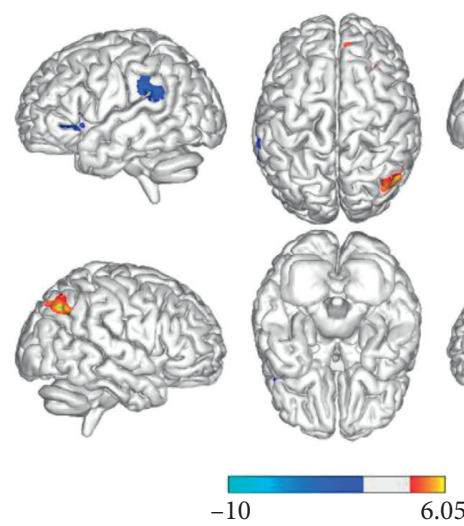

(e)
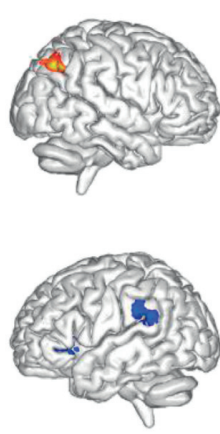

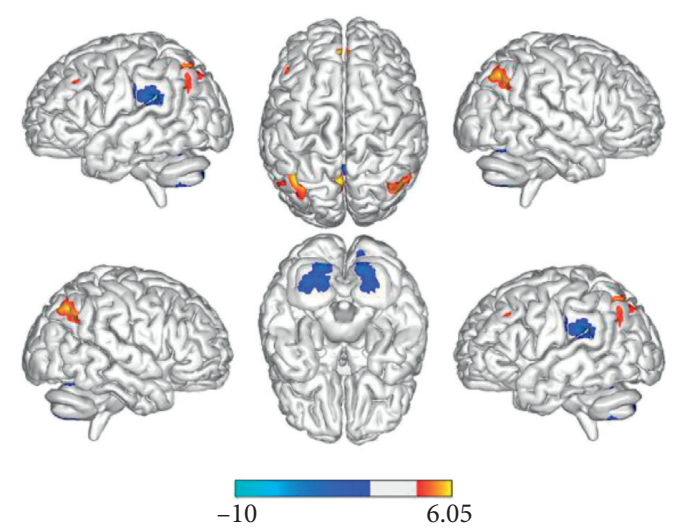

(d)
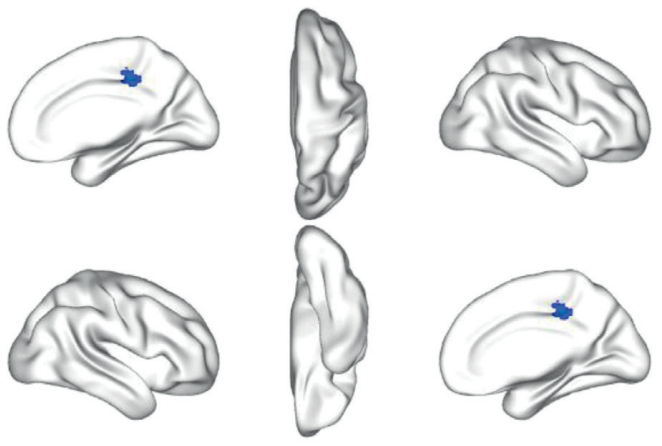

$-5.04$

(f)

FIGURE 3: Regions showing altered FC between the pre-PD group and the post-PD group (voxel $P<0.001$, FWE correction with cluster $P<0.05$ ). Left posterior cerebellar lobe (a), left middle temporal gyrus (b), bilateral precuneus (c), left middle frontal gyrus (d), right middle frontal gyrus (e), and bilateral paracentral lobule (f) as areas of interest, and FC analysis was performed by the seed-based approach; regions showing increased FC in red and decreased FC in blue; L, left hemispheres; R, right hemispheres; FWE, multiple comparisons of the familywise error (voxel $P<0.001$, cluster $P<0.05$ ); FC, functional connectivity; pre-PD, before DBS; post-PD, one day after DBS; PD, Parkinson's disease; DBS, deep brain stimulation.

validate this hypothesis. In summary, MLE can cause a reduction in the activity and connectivity of brain regions in the prefrontal cortex and DMN. Furthermore, there was increased FC of the precuneus and MFG with the following areas, including bilateral lingual gyrus, bilateral cerebellum, and right SMA. The lingual gyrus is a part of the occipital cortex and is involved in processing visual information [22]. However, after DBS, the visuospatial ability of patients decreased. We hypothesize that its enhanced connection to the DMN possibly represents a compensatory effect on visual processing in PD; however, further research is required. The cerebellum has an essential role in coordinating and controlling motion, and it is also a key area of the CTC. The decreased ReHo in the post-PD group might indicate partial damage to the CTC circuit after surgery. We speculate that in the microlesion stage, compared to the damage to the functioning of the CTC circuit, the damage caused by electrode implantation has a more significant compensatory effect on the function of the basal ganglia-thalamocortical circuit, ultimately causing an overall improvement in the patients' symptoms.
4.1. Limitations. First of all, this study was a preliminary study on the neural activity in the microlesion stage and the sample size is relatively small, so the sample size needs to be expanded for further study. Secondly, the period of postoperative microlesion is a phase of gradual change. This study did not conduct multiple assessments during the period of microlesion to better understand the changes in patients' symptoms. Finally, in this study, the effect of levodopa accumulation on the scale and resting-state data collection could not be completely eliminated even after withdrawal of anti-Parkinson/s disease drugs for 12 hours.

\section{Conclusion}

In conclusion, compared to HCs, alterations in DMN and visual association cortex areas of PD patients in this study further validated previous studies. The implantation of individual DBS electrodes changed the activity of the basal ganglia-thalamocortical circuit, prefrontal cortex, the DMN, and the CTC circuit and induced abnormal FC in the lingual gyrus, cerebellum, prefrontal cortex, and DMN in PD 
patients. However, the mechanism of microlesion remains to be further studied.

\section{Data Availability}

The relevant data used in this study are available from the corresponding author upon request.

\section{Conflicts of Interest}

The authors declare that there are no conflicts of interest.

\section{Authors' Contributions}

Bei Luo and Yue Lu contributed equally to this paper.

\section{Acknowledgments}

This study was supported by a subtopic of the 13th Five-Year National Key Research and Development Plan (No. 2016YFC0105901NNZ).

\section{References}

[1] D. J. Lee, C. S. Lozano, R. F. Dallapiazza, and A. M. Lozano, "Current and future directions of deep brain stimulation for neurological and psychiatric disorders," Journal of Neurosurgery, vol. 131, no. 2, pp. 333-342, 2019.

[2] A. M. Lozano, N. Lipsman, H. Bergman et al., "Deep brain stimulation: current challenges and future directions," Nature Reviews Neurology, vol. 15, no. 3, pp. 148-160, 2019.

[3] A. Mikos, D. Bowers, A. M. Noecker et al., "Patient-specific analysis of the relationship between the volume of tissue activated during DBS and verbal fluency," NeuroImage, vol. 54, no. Suppl 1, pp. S238-S246, 2011.

[4] D. Lee, R. Dallapiazza, P. De Vloo, and A. Lozano, "Current surgical treatments for parkinson's disease and potential therapeutic targets," Neural Regeneration Research, vol. 13, no. 8, pp. 1342-1345, 2018.

[5] W. Thevathasan, B. Debu, T. Aziz et al., "Pedunculopontine nucleus deep brain stimulation in parkinson's disease: a clinical review," Movement Disorders, vol. 33, no. 1, pp. 10-20, 2018.

[6] C. A. Edwards, A. Kouzani, K. H. Lee, and E. K. Ross, "Neurostimulation devices for the treatment of neurologic disorders," Mayo Clinic Proceedings, vol. 92, no. 9, pp. 1427-1444, 2017.

[7] S. Holiga, K. Mueller, H. E. Möller et al., "Resting-state functional magnetic resonance imaging of the subthalamic microlesion and stimulation effects in parkinson's disease: indications of a principal role of the brainstem," NeuroImage: Clinical, vol. 9, pp. 264-274, 2015.

[8] R. Jech, K. Mueller, D. Urgošík et al., "The subthalamic microlesion story in parkinson's disease: electrode insertionrelated motor improvement with relative cortico-subcortical hypoactivation in fMRI," PLoS One, vol. 7, no. 11, Article ID e49056, 2012.

[9] C. Granziera, C. Pollo, H. Russmann et al., "Sub-acute delayed failure of subthalamic DBS in parkinson's disease: the role of micro-lesion effect," Parkinsonism \& Related Disorders, vol. 14, no. 2, pp. 109-113, 2008.

[10] G. Costentin, S. Derrey, E. Gérardin et al., "White matter tracts lesions and decline of verbal fluency after deep brain stimulation in parkinson's disease," Human Brain Mapping, vol. 40, no. 9, pp. 2561-2570, 2019.

[11] P. J. Nathan, K. L. Phan, C. J. Harmer, M. A. Mehta, and E. T. Bullmore, "Increasing pharmacological knowledge about human neurological and psychiatric disorders through functional neuroimaging and its application in drug discovery," Current Opinion in Pharmacology, vol. 14, pp. 54-61, 2014.

[12] R. A. Poldrack and M. J. Farah, "Progress and challenges in probing the human brain," Nature, vol. 526, no. 7573, pp. 371-379, 2015.

[13] S. Lui, X. J. Zhou, J. A. Sweeney, and Q. Gong, "Psychoradiology: the frontier of neuroimaging in psychiatry," Radiology, vol. 281, no. 2, pp. 357-372, 2016.

[14] Y. Zang, T. Jiang, Y. Lu, Y. He, and L. Tian, "Regional homogeneity approach to fMRI data analysis," NeuroImage, vol. 22, no. 1, pp. 394-400, 2004.

[15] Y. Liu, M. Li, H. Chen et al., "Alterations of regional homogeneity in parkinson's disease patients with freezing of gait: a resting-state fMRI study," Frontiers in Aging Neuroscience, vol. 11, p. 276, 2019.

[16] Y. Yue, Y. Jiang, T. Shen, J. Pu, H.-Y. Lai, and B. Zhang, “ALFF and ReHo mapping reveals different functional patterns in early-and late-onset parkinson's disease," Frontiers in $\mathrm{Neu}$ roscience, vol. 14, p. 141, 2020.

[17] D. L. Harrington, Q. Shen, G. N. Castillo et al., "Aberrant intrinsic activity and connectivity in cognitively normal parkinson's disease," Frontiers in Aging Neuroscience, vol. 9, p. 197, 2017.

[18] J. Li, Y. Yuan, M. Wang et al., "Alterations in regional homogeneity of resting-state brain activity in fatigue of parkinson's disease," Journal of Neural Transmission, vol. 124, no. 10, pp. 1187-1195, 2017.

[19] Z. Li, A. Kadivar, J. Pluta, J. Dunlop, and Z. Wang, "Test-retest stability analysis of resting brain activity revealed by blood oxygen level-dependent functional MRI," Journal of Magnetic Resonance Imaging, vol. 36, no. 2, pp. 344-354, 2012.

[20] A. J. Hughes, S. E. Daniel, L. Kilford, and A. J. Lees, “Accuracy of clinical diagnosis of idiopathic parkinson's disease: a clinico-pathological study of 100 cases," Journal of Neurology, Neurosurgery \& Psychiatry, vol. 55, no. 3, pp. 181-184, 1992.

[21] K. Witt, C. Daniels, J. Reiff et al., "Neuropsychological and psychiatric changes after deep brain stimulation for parkinson's disease: a randomised, multicentre study," The Lancet Neurology, vol. 7, no. 7, pp. 605-614, 2008.

[22] A. Tessitore, F. Esposito, C. Vitale et al., "Default-mode network connectivity in cognitively unimpaired patients with parkinson disease," Neurology, vol. 79, no. 23, pp. 2226-2232, 2012.

[23] O. Lucas-Jiménez, N. Ojeda, J. Peña et al., "Altered functional connectivity in the default mode network is associated with cognitive impairment and brain anatomical changes in parkinson's disease," Parkinsonism \& Related Disorders, vol. 33, pp. 58-64, 2016.

[24] Y. Hou, J. Yang, C. Luo et al., "Dysfunction of the default mode network in drug-naïve parkinson's disease with mild cognitive impairments: a resting-state fMRI study," Frontiers in Aging Neuroscience, vol. 8, p. 247, 2016.

[25] Y. Abe, T. Kachi, T. Kato et al., "Occipital hypoperfusion in parkinson's disease without dementia: correlation to impaired cortical visual processing," Journal of Neurology, Neurosurgery \& Psychiatry, vol. 74, no. 4, pp. 419-422, 2003.

[26] H. Matsui, K. Nishinaka, M. Oda et al., "Hypoperfusion of the visual pathway in parkinsonian patients with visual 
hallucinations," Movement Disorders, vol. 21, no. 12, pp. 2140-2144, 2006.

[27] J. B. Rowe and H. R. Siebner, "The motor system and its disorders," NeuroImage, vol. 61, no. 2, pp. 464-477, 2012.

[28] B. Haslinger, P. Erhard, N. Kämpfe et al., "Event-related functional magnetic resonance imaging in parkinson's disease before and after levodopa," Brain, vol. 124, no. 3, pp. 558-570, 2001.

[29] A. Kikuchi, A. Takeda, T. Kimpara et al., "Hypoperfusion in the supplementary motor area, dorsolateral prefrontal cortex and insular cortex in parkinson's disease," Journal of the Neurological Sciences, vol. 193, no. 1, pp. 29-36, 2001.

[30] S. Kübel, K. Stegmayer, T. Vanbellingen, S. Walther, and S. Bohlhalter, "Deficient supplementary motor area at rest: neural basis of limb kinetic deficits in parkinson's disease," Human Brain Mapping, vol. 39, no. 9, pp. 3691-3700, 2018.

[31] S. Casarotto, F. Turco, A. Comanducci et al., "Excitability of the supplementary motor area in parkinson's disease depends on subcortical damage," Brain Stimulation, vol. 12, no. 1, pp. 152-160, 2019.

[32] M. Samuel, A. O. Ceballos-Baumann, N. Turjanski et al., "Pallidotomy in parkinson's disease increases supplementary motor area and prefrontal activation during performance of volitional movements an H2(15)O PET study," Brain, vol. 120, no. 8, pp. 1301-1313, 1997.

[33] R. G. Burciu and D. E. Vaillancourt, "Imaging of motor cortex physiology in parkinson's disease," Movement Disorders, vol. 33, no. 11, pp. 1688-1699, 2018.

[34] S. J. Kann, C. Chang, P. Manza, and H.-C. Leung, "Akinetic rigid symptoms are associated with decline in a cortical motor network in parkinson's disease," NPJ Parkinson's Disease, vol. 6 , no. 1, p. 19, 2020.

[35] S. Pietracupa, A. Suppa, N. Upadhyay et al., "Freezing of gait in parkinson's disease: gray and white matter abnormalities," Journal of Neurology, vol. 265, no. 1, pp. 52-62, 2018.

[36] B. Luo, Y. Lu, C. Qiu et al., "Altered spontaneous neural activity and functional connectivity in parkinson's disease with subthalamic microlesion," Frontiers in Neuroscience, vol. 15, Article ID 699010, 2021.

[37] R. Lefaucheur, S. Derrey, O. Martinaud et al., "Early verbal fluency decline after STN implantation: is it a cognitive microlesion effect?" Journal of the Neurological Sciences, vol. 321, no. 1-2, pp. 96-99, 2012.

[38] M. S. Okun, H. H. Fernandez, S. S. Wu et al., "Cognition and mood in parkinson's disease in subthalamic nucleus versus globus pallidus interna deep brain stimulation: the COMPARE trial," Annals of Neurology, vol. 65, no. 5, pp. 586-595, 2009.

[39] L. J. Michalski, C. H. Demers, D. A. A. Baranger et al., "Perceived stress is associated with increased rostral middle frontal gyrus cortical thickness: a family-based and discordant-sibling investigation," Genes, Brain and Behavior, vol. 16, no. 8, pp. 781-789, 2017.

[40] M. Koenigs and J. Grafman, "The functional neuroanatomy of depression: distinct roles for ventromedial and dorsolateral prefrontal cortex," Behavioural Brain Research, vol. 201, no. 2, pp. 239-243, 2009.

[41] E. K. Miller and J. D. Cohen, "An integrative theory of prefrontal cortex function," Annual Review of Neuroscience, vol. 24, no. 1, pp. 167-202, 2001. 